\title{
Desarrollo de un cuestionario de evaluación de la competencia docente en línea
}

\section{(Development of an assessment questionnaire for on- line teaching competency)}

\author{
Edna Luna Serrano \\ Alma Delia Hernández Villafaña \\ Universidad Autónoma de Baja California, UABC (México)
}

DOI: http://dx.doi.org/10.5944/ried.23.2.27084

\section{Cómo referenciar este artículo:}

Luna Serrano, E., y Hernández Villafaña, A. D. (2020). Desarrollo de un cuestionario de evaluación de la competencia docente en línea. RIED. Revista Iberoamericana de Educación a Distancia, 23(2), pp. 307-328. doi: http:// dx.doi.org/10.5944/ried.23.2.27084

\section{Resumen}

Evaluar la docencia con el fin de mejorar la calidad de la enseñanza implica reconocer su complejidad y contar con instrumentos de evaluación válidos que orienten hacia la mejora. El objetivo de este artículo es reportar el proceso de investigación que permitió desarrollar un cuestionario de evaluación formativa de la docencia en línea con base en la opinión de los estudiantes. Los participantes fueron seis expertos en el diseño e implementación de cursos en línea y un total de 2821 estudiantes universitarios. El método consistió en dos etapas: 1) Desarrollo del instrumento a través de fundamentación teórica sobre los elementos de la competencia docente en línea susceptibles de ser evaluados por estudiantes; elaboración de la matriz de especificación del cuestionario; validación de la matriz por el juicio de expertos y estudio piloto; 2) Aportación de evidencias de confiabilidad y validez incluyó la aplicación del cuestionario; cálculo de estadísticos descriptivos; análisis de unidimensionalidad por medio del modelo Rasch; determinación de la confiabilidad de los puntajes; análisis factorial exploratorio y confirmatorio. Se demostró que 28 reactivos evalúan el mismo constructo y se confirmaron dos factores: previsión del proceso de enseñanza y aprendizaje; y conducción y evaluación de la experiencia de enseñanza y aprendizaje; una varianza total de $70.01 \%$ y alpha ordinal de .998; un índice de ajuste RMSEA $=.08, \mathrm{SRMR}=.03, \mathrm{CFI}=.91$. Se concluye que los resultados corroboraron el fundamento teórico del instrumento con características psicométricas aceptables, por lo que se recomienda su aplicación.

Palabras clave: educación a distancia; evaluación formativa; evaluación del profesor; retroalimentación; calidad de la enseñanza. 


\begin{abstract}
Evaluating teaching to improve the quality of education implies to recognize its complexity and to count with valid assessment instruments that lead to improvement. The objective of this article is to report the research process that allowed the development of a formative assessment questionnaire of on-line teaching based on the opinion of students. The participants were six experts on the design and implementation of on-line courses, and a total of 2821 university students. The method consisted of two stages: 1) Development of the instrument through theoretical background regarding the elements of the on-line teaching competencies that are susceptible of being evaluated by students; the making of the questionnaire specification matrix; matrix validation by experts judgment and pilot study; 2) Contribution of confidence evidence and validity included the application of the questionnaire; descriptive statistical calculations; unidimensional analysis through the Rasch model; establishment of the scores reliability; exploratory and confirmatory factorial analysis. It was demonstrated that 28 items evaluate the same construct and 2 factors were confirmed: forecast of the teaching-learning process and the conduction and evaluation of the teaching and learning experience; a total variance of $0.01 \%$ and alpha ordinal of 0.998 ; and adjustment index RMSEA=0.08; $\mathrm{SRMR}=0.03, \mathrm{CF} 1=0.91$. It was concluded that the results corroborated the instrument's theoretical background with acceptable psychometric characteristics so that its application is recommended.
\end{abstract}

Keywords: distance education; formative evaluation; assessment teacher; feedback; quality of teaching.

La evaluación de la docencia se asume como una práctica social con implicaciones de carácter público y privado, y repercusiones para la sociedad, las instituciones y los docentes (Red Iberoamericana de Investigadores sobre la Evaluación de la Docencia [RIIED], 2008). Requiere ser coherente con la filosofía institucional, con el modelo pedagógico que sustenta la práctica docente y con las condiciones particulares del contexto donde se realiza la evaluación, solo así, se logrará que los resultados de la evaluación sean útiles para mejorar la docencia.

La evaluación docente con base en los cuestionarios de opinión de los estudiantes es la estrategia de evaluación más empleada en las instituciones de educación superior (IES) de Norteamérica, Europa y Asia (Hornstein, 2017; Smith y Kubacka, 2017). Los principales propósitos relacionados a su uso aluden a la rendición de cuentas sobre la calidad de la enseñanza, a la toma de decisiones administrativas y a la retroalimentación de los maestros para mejorar los procesos de enseñanza (Nasser-Abu, 2107).

Por lo general, los cuestionarios integran los componentes de la enseñanza asociados a la efectividad docente susceptibles a la observación y juicio de los estudiantes (Darwin, 2017). Desde la década de los años noventa se observaron las limitaciones de incluir únicamente las dimensiones asociadas a la efectividad docente en los cuestionarios y la necesidad de incorporar los cambios contextuales propios 
de cada situación educativa, en concreto, los planes de estudio por competencias y la educación a distancia (Benton y Cashin, 2014). En este sentido, se espera congruencia entre el instrumento de evaluación con la modalidad de enseñanza y modelo pedagógico que distingue a la práctica docente (validez de contenido).

Una particularidad de la enseñanza en línea es que tanto los materiales de estudio como la interacción entre docentes y estudiantes se realiza fundamentalmente a través de internet (García-Aretio, 2014). Esta circunstancia impone una compleja interacción entre el contenido, la pedagogía y la tecnología que los instrumentos de evaluación deben operacionalizar.

La importancia de la evaluación de la enseñanza en línea ha sido ampliamente documentada. Cabe precisar que la evaluación se ha orientado a los factores directamente relacionados con la calidad de la educación virtual, donde las dimensiones frecuentes son: el contexto institucional, la infraestructura tecnológica, los estudiantes y el docente (Luna, Ponce, Cordero y Cisneros-Cohernour, 2018). En el mismo sentido, los estrechamente relacionados con los cursos, a saber, el diseño, el contenido, el docente y el sistema de apoyo para estudiantes y docentes (McClary, 2013). Sin embargo, la investigación no ha centrado su interés en la actividad docente. Así, no se ha prestado suficiente atención al desarrollo y validación de instrumentos de evaluación de la docencia orientados a la mejora de la actividad. El objetivo de este artículo es reportar el proceso de investigación que permitió desarrollar un cuestionario de evaluación de la competencia docente en línea con base en la opinión de los estudiantes en educación superior.

La evaluación de la docencia es una tarea compleja, en específico en la docencia en línea se advierte la diversidad de roles asignados al docente, mismos que dependen de las características y tamaño de la institución y pueden ser desempeñados por más de una persona o figuras (planificadores de programas, responsables de guiar el aprendizaje, tutores, etc.). No obstante, por lo general la figura docente involucra la responsabilidad de conducir el curso, de tener presencia con los estudiantes proporcionando retroalimentación puntual y personalizada con cada estudiante y de evaluar los aprendizajes (McClary, 2013).

En la búsqueda de las cualidades esenciales que definen a la docencia en línea se han planteado diferentes aproximaciones. Una de las propuestas más aceptadas es el modelo TPCK [Technological Pedagogical Content Knowledge] (Mishra y Koehler, 2006), que propone la articulación de los componentes contenido, pedagogía y tecnología analizados en diadas: conocimiento pedagógico del contenido (PCK), conocimiento tecnológico y de contenido (TCK), conocimiento tecnológico pedagógico (TPK); y la integración en conocimiento tecnológico, pedagógico y de contenido (TPCK). A partir de este modelo, García-Aretio (2014) planteó como elementos que definen a la docencia de calidad en línea: el compromiso disciplinar (contenidos); el compromiso pedagógico/didáctico que incluye la competencia metodológica, competencia comunicacional, competencia tutorial, competencia evaluadora; el compromiso tecnológico y el compromiso investigador e innovador. 
Desde una perspectiva constructiva, social y comunicativa; Mauri y Onrubia (2008) plantearon las competencias de diseño de la interactividad tecnológica, diseño de la interactividad pedagógica y desarrollo o uso tecnopedagógico. El Modelo de Evaluación de Competencias Docentes en Línea (MECDL) de García-Cabrero et al. (2018) operacionaliza mediante competencias e indicadores los planteamientos teóricos y conceptuales derivados de modelos sustentados en el constructivismo socio-cultural, el constructivismo social y el conectivismo. En particular del Modelo de Comunidad de Indagación (CoI), incluye elementos relacionados con las categorías Presencia Docente, Presencia Cognitiva y Presencia Social (Garrison, Anderson y Archer, 2000), asimismo la Presencia Emocional (Cleveland-Innes y Campbell, 2012). Además, del Modelo Conversacional de Laurillard (2002) lo alusivo a la conversación entre el docente y los alumnos, los planteamientos derivados de los modelos de diseño instruccional para ambientes virtuales, tales como proporcionar retroalimentación oportuna y facilitar el desarrollo paulatino de la autonomía del alumno; tanto como los modelos flexibles de enseñanza en lo relativo a adaptar las actividades de acuerdo con las necesidades individuales o grupales de los alumnos. Incluye la dimensión previsión del proceso de enseñanza-aprendizaje (con 5 competencias y 27 indicadores); conducción del proceso de enseñanza-aprendizaje (con 3 competencias y 40 indicadores); y valoración del impacto del proceso enseñanza-aprendizaje (con una competencia y 6 indicadores). Con la característica de que es un modelo validado para las IES mexicanas. Además, se formuló como un marco de referencia conceptual para orientar la práctica docente, su evaluación y formación del profesorado virtual.

\section{Evaluación docente en línea con base en los cuestionarios de opinión de los estudiantes}

Las dimensiones de evaluación utilizadas en los cuestionarios de evaluación de docencia en línea por los alumnos reflejan la aproximación teórica sobre la enseñanza en línea que se privilegia. Entre los cuestionarios publicados con evidencias psicométricas se destacan los siguientes.

En Estados Unidos, el diseñado por Bangert (2008) "Student Evaluation of Online Teaching effeciveness" (SEOTE) fue desarrollado con el propósito de probar la aplicabilidad a la enseñanza en línea de los Siete Principios de Buenas Prácticas en la Educación de Pregrado de Chickering y Gamson (1987), que se fundamentaron en la aproximación constructivista del aprendizaje, y son los siguientes: 1) promover la interacción profesor-estudiante, 2) alentar la cooperación entre los estudiantes, 3) alentar el aprendizaje activo, 4) proporcionar retroalimentación oportuna, 5) enfatizar el tiempo de las tareas, 6) comunicar altas expectativas y 7) respetar los diversos talentos y formas de aprendizaje de los estudiantes. El SEOTE incluye las dimensiones: interacción profesor-estudiante; aprendizaje activo; tiempo de respuesta y cooperación entre estudiantes con un total de 23 reactivos. La adaptación 
del SEOTE al contexto canadiense arrojó tres factores: instructor, interacción y curso específico con 24 reactivos (Ravenscroft, Luhanga y King, 2017). También, Baldwin y Trespalacions (2017) estudiaron 28 instrumentos con el fin de identificar cuáles son los Principios de Buenas Prácticas más utilizados. En orden descendente se ubicaron: promover la interacción profesor-estudiante $(85 \%)$, alentar la cooperación entre estudiantes (75\%) y alentar el aprendizaje activo (57\%). Por el contrario, los menos empleados resultaron: proporcionar retroalimentación oportuna (29\%), comunicar altas expectativas (14\%) y el tiempo de las tareas (4\%).

El cuestionario de Arbaugh et al. (2008) se desarrolló con base en el modelo conceptual para el aprendizaje en línea de CoI que se fundamenta en una perspectiva constructivista-colaborativa. Por lo tanto, operacionaliza los componentes centrales de CoI: la presencia social, definida como la habilidad de los participantes de proyectar características personales y aparecer ante los otros como personas reales, engloba tres categorías (comunicación abierta, cohesión del grupo y proyección personal afectiva) lo que facilita el establecimiento de un entorno de apoyo que genera comodidad y seguridad para expresar ideas en un contexto colaborativo; la presencia cognitiva, alude al grado en el cual los participantes son capaces de construir significados a través de comunicación sustantiva, incorpora las categorías desencadenar eventos, exploración de ideas, integración y resolución; la presencia docente, incluye las categorías de diseño instruccional, facilitación de la discusión y colaboración e instrucción directa (Garrison et al., 2000). Los análisis psicométricos fundamentaron el constructo presencia docente con dos factores: diseño y organización del curso, así como comportamiento del instructor durante el curso, con una solución de 34 reactivos. La adaptación de este cuestionario al idioma turco corroboró su estructura (Olpak y Kilic, 2018). Los instrumentos antes mencionados se distinguen porque fueron desarrollados en idioma inglés, para ser utilizados de forma institucional y cuentan con evidencias de validez. Cabe precisar que no se encontraron publicados instrumentos desarrollados en español.

\section{MÉTODO}

\section{Participantes}

Se contó con la colaboración de dos grupos de participantes: expertos en el diseño e implementación de cursos en línea y estudiantes.

Grupo de expertos. El grupo se conformó por seis especialistas adscritos a distintas universidades mexicanas. La selección de los participantes se realizó con base en su experiencia y trayectoria académica. Así mismo, se procuró incluir expertos de diversas especialidades en el campo de la educación en línea. Por ello, se incluyó un experto con más de 25 años de experiencia en docencia, gestión e investigación de modelos educativos en línea; un especialista en innovación educativa y ambientes e-learning; uno en diseño instruccional y desarrollo de software 
educativo; en formación y evaluación de la docencia en línea; otro con experiencia en la administración de programas en línea a nivel institucional y un especialista en psicometría.

Estudiantes. Se buscó representatividad de la población de estudiantes inscritos en los cursos en línea de los programas de las diferentes áreas de conocimiento de la universidad. Así, por un lado, en el estudio piloto, los puntajes corresponden a una muestra autoseleccionada de 296 estudiantes distribuidos en los tres campus de la universidad; y, por otro lado, en la etapa de aportación de evidencias de validez los puntajes analizados pertenecen a una muestra autoseleccionada de 2525 estudiantes (quienes representan el 37.25\%) de la población que se encontraba inscrita en el segundo periodo semestral de 2017 en los cursos en línea de la Universidad Autónoma de Baja California (UABC) en México. El 54\% de sexo femenino y $46 \%$ de masculino, con una media aritmética de 20 años. La distribución por áreas del conocimiento de los estudiantes fue: ingeniería y tecnología $29 \%$; ciencias agropecuarias $4 \%$; ciencias de la salud 11\%; ciencias naturales y exactas $1 \%$, educación y humanidades $38 \%$ y ciencia sociales y administrativas $17 \%$.

\section{Diseño}

Corresponde a un estudio transversal de tipo descriptivo.

\section{Materiales}

Modelo de Evaluación de Competencias Docentes en Línea [MECDL] (GarcíaCabrero et al., 2018). El MECDL contiene tres dimensiones: previsión del proceso enseñanza-aprendizaje, conducción del proceso enseñanza-aprendizaje y valoración del impacto del proceso enseñanza-aprendizaje.

Formulario de valoración de los reactivos. Incluyó una revisión general del constructo, análisis de contenido de cada reactivo, y valoración de aceptar, modificar o eliminar un reactivo (Sireci y Faulker-Bond, 2014).

Formulario de validación para los jueces. El formulario se utilizó de forma impresa y en hojas de cálculo de Google. Los criterios establecidos para la validación de los ítems fueron los que, comúnmente, se usan en este tipo de trabajos: claridad, relevancia y congruencia. Para la validación de las competencias, el criterio fue el grado de suficiencia de los ítems y la inclusión de los ítems necesarios para evaluar cada competencia. Se empleó una escala del $1 \mathrm{al} 4$ para todos los casos, donde 1 representa la mínima valoración y 4 la máxima. Además, a cada experto se le otorgó el equipo de cómputo que requería para realizar el proceso de validación. 


\section{Procedimiento}

El trabajo se desarrolló en dos etapas que, a continuación, se describen.

\section{Etapa 1. Desarrollo del cuestionario}

Fase 1. Fundamentación teórica sobre los elementos de la competencia docente en línea susceptibles de ser evaluados por estudiantes. Con el fin de analizar la teoría sustantiva que fundamenta la competencia docente en línea y su evaluación, se realizó una búsqueda de modelos de evaluación e instrumentos de evaluación de la docencia en línea con base en la opinión de los estudiantes de 2000 a 2018, en las bases de datos Elsevier y Proquest, Eric, Scopus, SAGE, EBSCO, Google Académico y Google.

Fase 2. Elaboración de la matriz de especificación del Cuestionario de Evaluación de la Competencia Docente en Línea (CECDL). La matriz se organizó de acuerdo con la estructura del MECDL en dimensiones con sus respectivas competencias, de los indicadores se seleccionaron los valorados como apropiados para ser evaluados por los estudiantes, a partir de los cuales se elaboraron los reactivos.

Fase 3. Validación de la matriz de especificación del cuestionario. Se llevó a cabo en dos momentos: 1) El psicómetra valoró los componentes de la matriz (competencias, indicadores y reactivos) con base en el formulario de valoración de los reactivos. Posteriormente, el resultado se sometió al juicio de los expertos en enseñanza en línea. 2) La sesión de validación se realizó a manera de seminario, a cada experto se le entregó el formato de validación de forma impresa, y en formato electrónico en hojas de cálculo de Google. Para ello, se dispuso del equipo de cómputo necesario para cada participante. Se valoraron tres elementos: 1) claridad del reactivo, se refiere al grado en que comunica de manera objetiva el enunciado; 2) relevancia del reactivo, es el grado en que puede ser contestado por el estudiante; 3) congruencia, alude al grado en que los reactivos se corresponden lógicamente con la competencia a la que pertenecen.

Primero se valoró la dimensión de Previsión, seguida de Conducción y, al final, Valoración. Los expertos evaluaron de manera individual y registraron en la hoja de cálculo el puntaje otorgado a cada reactivo de forma sincrónica. Además, se tuvo la posibilidad de agregar comentarios sobre los reactivos. Por último, se procedió a la revisión grupal de los reactivos con una puntuación por abajo de 2.5 conforme a la escala, las modificaciones se acordaron por consenso. De esta manera, se buscó el acuerdo inter-subjetivo del grupo de expertos en relación con los ajustes a realizar (Bakieva, Jornet, González-Such y Leyva, 2018).

Fase 4. Estudio piloto. El cuestionario se envió a través de la Coordinación del Centro de Educación Abierta y a Distancia a 500 estudiantes inscritos en los cursos en línea del primer periodo semestral de 2017. Se realizaron análisis descriptivos 
(mediana, media y desviación estándar de los ítems); se calcularon los índices de consistencia interna (alfa ordinal) y el coeficiente de correlación punto biserial.

\section{Etapa 2. Aportación de evidencias confiabilidad y validez}

Fase 1. Aplicación del cuestionario. El cuestionario fue capturado en una plataforma diseñada ex profeso y enviado vía correo electrónico institucional a 6777 estudiantes (todos los estudiantes inscritos en los cursos en línea que ofertó la UABC durante el segundo periodo semestral de 2017).

Fase 2. Integración de la base de datos y análisis descriptivos. Los puntajes fueron integrados en una base de datos en el software Statistical Package for the Social Sciencies [SPSS] versión 21. Conforme al cuestionario, se establecieron 28 variables y el número de datos fueron 2525. Se obtuvieron los estadísticos descriptivos referente al número de casos, frecuencias, porcentajes, media aritmética y desviación estándar de los puntajes.

Fase 3. Análisis de unidimensionalidad. La unidimensionalidad fue determinada por medio del modelo Rasch por sus propiedades de invarianza y escala de intervalo (Tabatabaee-Yazdi, Motallebzadeh, Ashraf y Baghaei, 2018). Este análisis se realizó en el software Winsteps versión 3.81.0, obteniendo los índices de dificultad de los ítems y el ajuste de los datos al modelo.

Fase 4. Análisis de consistencia interna. El coeficiente de consistencia interna para medir la confiabilidad de los puntajes fue el alfa ordinal. Se determinó por medio del software RStudio con el paquete Psych. Las variables del cuestionario fueron ordinales, por lo tanto, se optó por las correlaciones policóricas dado que representan una estimación más precisa de confiabilidad (Domínguez-Lara, 2018).

Fase 5. Análisis factorial exploratorio (AFE). El AFE se realizó en el software SPSS con la finalidad de reducir el número de variables observadas en un número mínimo de factores (Verma, 2019). De la muestra se seleccionó una submuestra aleatoria aproximada al 50\% de los datos, conformada por 1278 datos con los cuales se calculó el AFE.

Fase 6. Análisis factorial confirmatorio (AFC). El AFC se ejecutó con el software $E Q S$ 6.1, este análisis se enfoca en la relación subyacente entre los factores latentes que dan evidencia de una teoría sobre estos factores (Finch y French, 2019). La submuestra fue de 1243 datos determinada en el software SPSS tal como se describe en el AFE.

\section{RESULTADOS}

El diseño del cuestionario se fundamentó en el MECDL (García-Cabrero et al., 2018) en virtud de la fundamentación teórica que lo sustenta y por haber sido desarrollado para el contexto de enseñanza de México. En este sentido, se consideró como el modelo hipotético. Por tanto, la primera matriz de especificaciones del 
cuestionario quedó integrada por las tres dimensiones, las nueve competencias del MECDL y 84 reactivos.

Validación de la matriz de especificación del cuestionario. Los comentarios de los expertos se concentraron en cuatro categorías: 1) se incluyen muchos ítems; 2) presenta contenido repetido entre las dimensiones; 3) uso de lenguaje especializado con términos técnicos difícil de comprender por los estudiantes; y 4) se sugiere seleccionar los ítems esenciales plausibles de ser evaluados por los estudiantes. Se revisaron todos los ítems y con base en el consenso, se procedió a eliminar los ítems considerados repetidos, a modificar la redacción eliminando los términos técnicos, e incluir solo los ítems cuyo contenido se considera esencial y factible de ser valorado por los estudiantes. La tabla 1 presenta un concentrado de los resultados del proceso de validación. El producto de esta fase fue la segunda versión del cuestionario que incluye 28 reactivos distribuidos en las tres dimensiones.

Tabla 1. Resultados del proceso de validación de contenido de la tabla de especificaciones

\begin{tabular}{|c|c|c|c|c|c|c|}
\hline & $\begin{array}{c}\text { Previsión del } \\
\text { proceso enseñanza- } \\
\text { aprendizaje }\end{array}$ & \multicolumn{3}{|c|}{$\begin{array}{l}\text { Conducción del } \\
\text { proceso enseñanza- } \\
\text { aprendizaje }\end{array}$} & \multicolumn{2}{|c|}{$\begin{array}{l}\text { Evaluación del } \\
\text { impacto del proceso } \\
\text { de enseñanza- } \\
\text { aprendizaje }\end{array}$} \\
\hline $\begin{array}{l}\text { Comentarios } \\
\text { principales } \\
\text { sobre los } \\
\text { ítems }\end{array}$ & $\begin{array}{l}\text { Muchos ítems. } \\
\text { Contenido repetido. } \\
\text { Ítems muy } \\
\text { especializados } \\
\text { (lenguaje técnico } \\
\text { y difícil para los } \\
\text { estudiantes). }\end{array}$ & \multicolumn{3}{|c|}{\begin{tabular}{|l|} 
Muchos ítems. \\
Contenido repetido con \\
previsión. \\
Îtems muy \\
especializados \\
(lenguaje técnico \\
y difícil para los \\
estudiantes). \\
Seleccionar ítems \\
esenciales que el \\
estudiante pueda \\
valorar.
\end{tabular}} & \multicolumn{2}{|c|}{$\begin{array}{l}\text { Muchos ítems. } \\
\text { Contenido repetido } \\
\text { con previsión o } \\
\text { Conducción. } \\
\text { Ítems muy } \\
\text { especializados } \\
\text { (lenguaje técnico } \\
\text { y difícil para los } \\
\text { estudiantes). } \\
\text { Seleccionar ítems que } \\
\text { el estudiante pueda } \\
\text { valorar. }\end{array}$} \\
\hline $\begin{array}{l}\text { Criterio } \\
\text { al que } \\
\text { corresponden } \\
\text { los ítems con } \\
\text { puntuación } \\
\text { menor a } 2.5\end{array}$ & $\mathrm{C}$ & $\mathrm{C}$ & $\mathrm{R}$ & Co & $\mathrm{C}$ & $\mathrm{S}$ \\
\hline $\begin{array}{l}\text { Ítems } \\
\text { revisados }\end{array}$ & 27 & \multicolumn{3}{|c|}{44} & \multicolumn{2}{|c|}{14} \\
\hline $\begin{array}{l}\text { Ítems } \\
\text { resultantes }\end{array}$ & 8 & \multicolumn{3}{|c|}{17} & \multicolumn{2}{|c|}{3} \\
\hline
\end{tabular}

Nota: C: claridad; R: relevancia; Co: congruencia; S: suficiencia. 
Fase 4. Estudio piloto. Los resultados derivados del estudio piloto fundamentaron continuar con la siguiente etapa. En la Etapa de Aportación de Evidencias de Confiabilidad y Validez, los puntajes proceden de 2525 estudiantes que contestaron completamente el cuestionario, equivalentes al $37.25 \%$ de la población inscrita en los cursos en línea que oferta la universidad.

Análisis de unidimensionalidad. La estimación de las propiedades del cuestionario arrojó que, de los 28 ítems, 27 registraron índices de ajuste estables del INFIT y del OUTFIT dado que se ubicaron entre el 0.5 al 1.5 (Linacre, 2002), con excepción del ítem 1 (Estableció la relación de la asignatura con el campo profesional) y el 22 (Realiza una evaluación diagnóstica al inicio de la asignatura), además éste registró el mayor índice de nivel de dificultad (ver tabla 2).

Tabla 2. Ajuste de los valores del análisis de unidimensionalidad

\begin{tabular}{|c|c|c|c|c|c|}
\hline Ítem & $\begin{array}{l}\text { Correlación } \\
\text { punto biserial }\end{array}$ & $\begin{array}{l}\text { Discriminación } \\
\text { del reactivo }\end{array}$ & $\begin{array}{l}\text { Dificultad del } \\
\text { reactivo }\end{array}$ & $\begin{array}{l}\text { Ajuste } \\
\text { cercano }\end{array}$ & $\begin{array}{l}\text { Ajuste } \\
\text { lejano }\end{array}$ \\
\hline 1 & 0.86 & 0.71 & 0.20 & 1.15 & 1.65 \\
\hline 2 & 0.91 & 0.93 & -0.56 & 1.00 & 1.29 \\
\hline 3 & 0.90 & 0.93 & -0.31 & 1.02 & 1.17 \\
\hline 4 & 0.91 & 0.79 & -0.49 & 1.26 & 1.39 \\
\hline 5 & 0.92 & 1.03 & -0.29 & 0.96 & 1.00 \\
\hline 6 & 0.88 & 0.75 & -0.38 & 1.25 & 1.56 \\
\hline 7 & 0.91 & 1.03 & -0.42 & 0.96 & 0.96 \\
\hline 8 & 0.90 & 1.00 & -0.49 & 1.01 & 1.01 \\
\hline 9 & 0.90 & 1.23 & -0.48 & 0.73 & 0.75 \\
\hline 10 & 0.90 & 1.16 & -0.24 & 0.81 & 0.81 \\
\hline 11 & 0.93 & 1.30 & -0.11 & 0.65 & 0.67 \\
\hline 12 & 0.90 & 1.15 & 0.21 & 0.80 & 0.83 \\
\hline 13 & 0.90 & 1.00 & 0.71 & 0.96 & 1.04 \\
\hline 14 & 0.91 & 1.11 & 0.43 & 0.85 & 0.93 \\
\hline 15 & 0.90 & 0.90 & 1.04 & 1.10 & 1.10 \\
\hline 16 & 0.91 & 1.07 & 0.39 & 0.93 & 0.94 \\
\hline 17 & 0.92 & 1.23 & -0.06 & 0.76 & 0.69 \\
\hline 18 & 0.92 & 1.20 & -0.09 & 0.78 & 0.80 \\
\hline 19 & 0.93 & 1.19 & 0.37 & 0.81 & 0.76 \\
\hline 20 & 0.92 & 1.20 & 0.29 & 0.80 & 0.73 \\
\hline 21 & 0.92 & 1.11 & 0.52 & 0.90 & 0.87 \\
\hline 22 & 0.80 & -0.48 & 1.68 & 2.35 & 2.40 \\
\hline 23 & 0.84 & 0.96 & -1.12 & 1.07 & 0.98 \\
\hline 24 & 0.91 & 1.02 & 0.51 & 0.96 & 0.98 \\
\hline
\end{tabular}




\begin{tabular}{|c|c|c|c|c|c|}
\hline Ítem & $\begin{array}{c}\text { Correlación } \\
\text { punto biserial }\end{array}$ & $\begin{array}{c}\text { Discriminación } \\
\text { del reactivo }\end{array}$ & $\begin{array}{c}\text { Dificultad del } \\
\text { reactivo }\end{array}$ & $\begin{array}{c}\text { Ajuste } \\
\text { cercano }\end{array}$ & $\begin{array}{c}\text { Ajuste } \\
\text { lejano }\end{array}$ \\
\hline 25 & 0.92 & 1.17 & 0.48 & 0.83 & 0.88 \\
\hline 26 & 0.92 & 1.01 & -1 & 1.00 & 1.02 \\
\hline 27 & 0.96 & 1.02 & -0.47 & 0.98 & 1.00 \\
\hline 28 & 0.95 & 1.21 & -0.32 & 0.76 & 0.75 \\
\hline
\end{tabular}

Análisis de consistencia interna. La consistencia interna (alfa ordinal) del cuestionario registró un índice de .998, la dimensión de Previsión del proceso de enseñanza-aprendizaje presentó un alfa ordinal de .967, la dimensión Conducción del proceso de enseñanza-aprendizaje de .986 y la dimensión Evaluación del impacto del proceso enseñanza-aprendizaje .953 (ver tabla 3).

Tabla 3. Índices de consistencia interna (alfa ordinal)

\begin{tabular}{|l|l|c|}
\hline & \multicolumn{2}{|c|}{ Alfa ordinal } \\
\hline \multirow{3}{*}{ Dimensión } & CECDL & .998 \\
\hline \multirow{2}{*}{} & Previsión & .997 \\
\cline { 2 - 3 } & Conducción & .986 \\
\cline { 2 - 3 } & Evaluación & .953 \\
\hline
\end{tabular}

Análisis factorial exploratorio (AFE). El AFE se realizó con el método de extracción de componentes principales, aplicándose rotación ortogonal Varimax con normalización Kaiser con coeficientes mayores a 0.40. Los factores fueron nombrados con base a las cargas factoriales y al modelo teórico que sustenta al cuestionario. Los resultados arrojan la agrupación de los datos en dos factores que explican el 70.01\% de la varianza. El Factor 1 coincide con la dimensión de Previsión del proceso de enseñanza-aprendizaje. Aquí se ubicaron los 8 ítems de esta dimensión, un ítem de Conducción de la experiencia de enseñanza-aprendizaje y otro ítem de Valoración del impacto del proceso de enseñanza-aprendizaje, por lo tanto, el Factor 1 se denominó con el mismo nombre. El Factor 2 se integró por la mayoría de los ítems de la dimensión de Conducción de la experiencia de enseñanzaaprendizaje y por los dos ítems restantes de Valoración del impacto del proceso de enseñanza-aprendizaje. Este Factor se denominó Conducción y Evaluación de la experiencia de enseñanza y aprendizaje (ver tabla 4). 
Tabla 4. Cargas factoriales por cada factor

\section{Ítem}

\begin{tabular}{|c|c|c|}
\hline \multirow{2}{*}{ Ítem } & \multicolumn{2}{|c|}{ Factores } \\
\hline & 1 & 2 \\
\hline 2. Estableció las metas de aprendizaje para la asignatura. & .764 & \\
\hline $\begin{array}{l}\text { 4. Estableció al inicio del curso un cronograma detallado de actividades (por } \\
\text { ejemplo, períodos de trabajo, fechas de entrega de tareas individuales y } \\
\text { grupales, exámenes). }\end{array}$ & .754 & \\
\hline $\begin{array}{l}5 \text { Acordó al inicio de la asignatura las reglas de interacción en medios } \\
\text { digitales sustentadas en valores universales. }\end{array}$ & .742 & \\
\hline $\begin{array}{l}\text { 3. Seleccionó materiales digitales pertinentes en contenido para las } \\
\text { actividades de aprendizaje. }\end{array}$ & .738 & \\
\hline $\begin{array}{l}\text { 7. Presentó en cada unidad instrucciones detalladas para su desarrollo (por } \\
\text { ejemplo, propósitos de las actividades, dinámicas de trabajo). }\end{array}$ & .721 & \\
\hline 23. Hace un correcto uso del lenguaje. & & \\
\hline 6. Definió las reglas de trabajo para evitar el plagio. & .693 & \\
\hline 26. Respeta los criterios de evaluación presentados al inicio del curso. & .690 & \\
\hline $\begin{array}{l}\text { 8. Incluye en las actividades el uso de Tecnologías de la Información y la } \\
\text { Comunicación (videos, wikis, blogs, aplicaciones web, software) acordes a } \\
\text { mis posibilidades de aprendizaje. }\end{array}$ & .686 & \\
\hline 1. Estableció la relación de la asignatura con el campo profesional. & .613 & \\
\hline $\begin{array}{l}\text { 15. Proporciona retroalimentación de forma personalizada a través de la } \\
\text { plataforma. }\end{array}$ & & .797 \\
\hline $\begin{array}{l}\text { 24. Mantiene comunicación continua con los estudiantes a través de los } \\
\text { medios de interacción de la plataforma. }\end{array}$ & & .773 \\
\hline $\begin{array}{l}\text { 25. Retroalimenta de forma oportuna el desarrollo de las actividades de } \\
\text { aprendizaje. }\end{array}$ & & .768 \\
\hline 21. Fomenta el sentido de bienestar en el grupo. & & .767 \\
\hline $\begin{array}{l}\text { 19. Orienta a los estudiantes cuando existen dudas o problemas con el manejo } \\
\text { de las Tecnologías de la Información y la Comunicación (por ejemplo, } \\
\text { aplicaciones web y la plataforma) que se utilizan en el curso. }\end{array}$ & & .746 \\
\hline 13. Aclara las dudas de forma oportuna. & & .744 \\
\hline 22. Realiza una evaluación diagnóstica al inicio de la asignatura. & & .736 \\
\hline $\begin{array}{l}\begin{array}{l}\text { 20. Fomenta que los estudiantes reflexionen sobre sus experiencias de } \\
\text { aprendizaje. }\end{array} \\
\end{array}$ & & .708 \\
\hline $\begin{array}{l}\text { 16. Estructura actividades de aprendizaje que propician la colaboración entre } \\
\text { los estudiantes. }\end{array}$ & & .707 \\
\hline $\begin{array}{l}\text { 14. Ajusta las actividades de aprendizaje de acuerdo con las necesidades de los } \\
\text { estudiantes. }\end{array}$ & & .691 \\
\hline $\begin{array}{l}\text { 12. Promueve la aplicación de los aprendizajes en la solución de problemas } \\
\text { reales. }\end{array}$ & & .633 \\
\hline 11. Promueve el desarrollo de las actitudes señaladas en la asignatura. & & .578 \\
\hline
\end{tabular}




\section{Ítem}

17. Utiliza materiales digitales en diversos formatos para enriquecer la experiencia de aprendizaje.

18. Verifica que los materiales digitales seleccionados para cada actividad de aprendizaje estén accesibles de forma oportuna.

28. Utiliza diversas estrategias para la evaluación del curso acordes con los objetivos de aprendizaje.

27. Realiza una evaluación final congruente con las actividades de aprendizaje realizadas durante el curso.

10. Facilita los materiales necesarios para desarrollar los conocimientos propuestos en la asignatura

9. Implementa actividades de aprendizaje acordes con los propósitos de la asignatura.

Análisis factorial confirmatorio ( $A F C$ ). El AFC fue configurado conforme al modelo del análisis anterior. Se utilizó el método de estimación de Máxima Verosimilitud (por sus siglas en ingles ML). La figura 1 muestra el esquema del modelo, los círculos representan los factores y los rectángulos las 28 variables. La flecha bidireccional simboliza la covarianza entre el factor 1 y 2 (variables latentes) y las flechas unidireccionales representan la influencia del factor o dimensión sobre el ítem (variable observada). 
E. Luna Serrano; A. D. Hernández Villafaña

DESARROLLO DE UN CUESTIONARIO DE EVALUACIÓN DE LA COMPETENCIA DOCENTE EN LÍNEA

Figura 1. Esquema del modelo del AFC

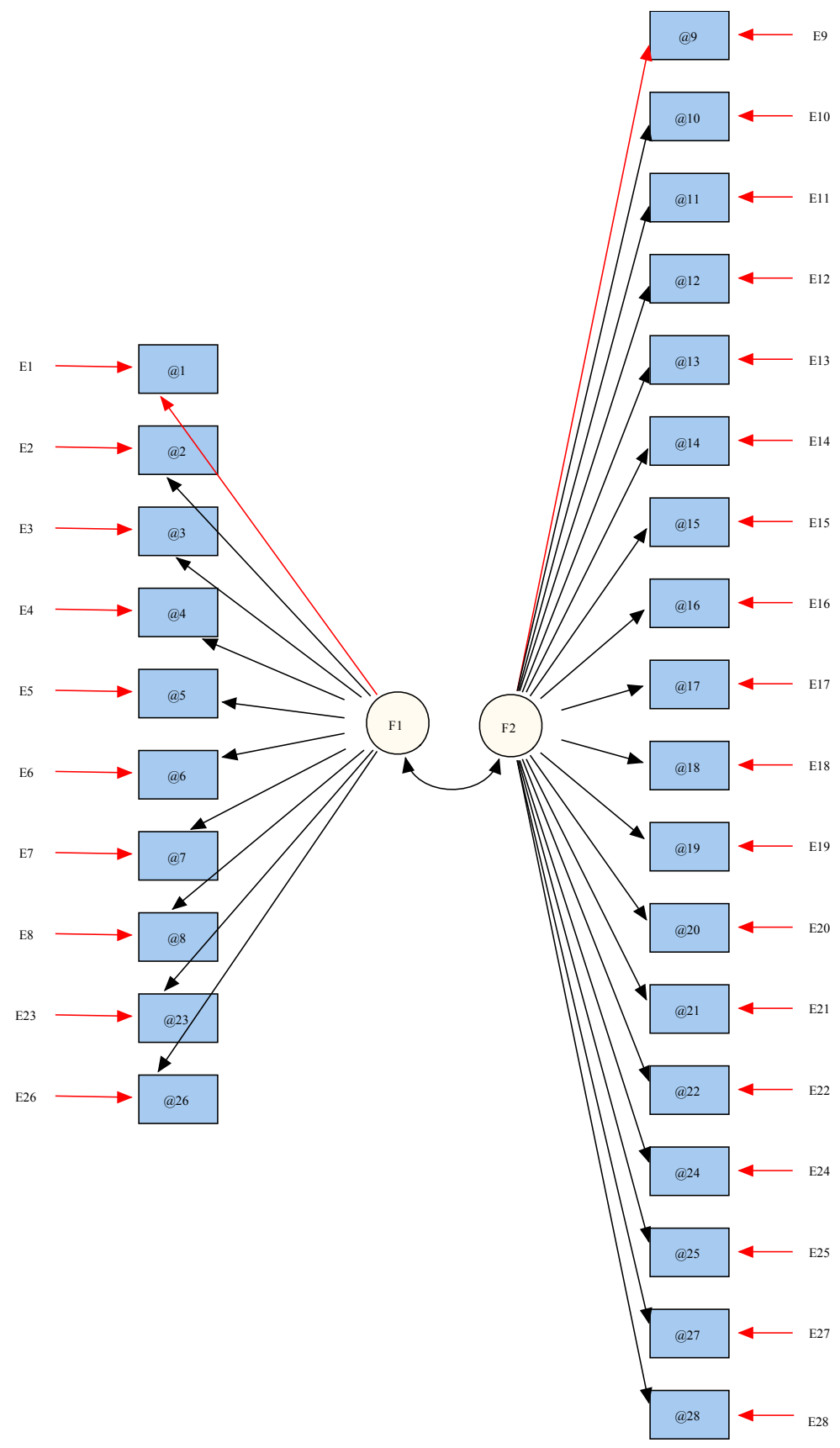


Los resultados del análisis, basado en el modelo de dos factores, se ajustan bien a los datos. Los índices reflejan que la raíz del residuo cuadrático promedio de aproximación (RMSEA) fue de .08, la raíz del residuo cuadrático promedio estandarizado (SRMR) fue .03. El índice de bondad de ajuste comparativo (CFI) es del orden .91 (ver tabla 5). Los índices de bondad de ajuste expuestos otorgan elementos que confirman el modelo propuesto.

Tabla 5. Índices de ajuste del modelo del Factor 1 y 2

\begin{tabular}{|l|c|c|}
\hline \multicolumn{1}{|c|}{ Indice } & Criterio & Modelo F1 y F2 \\
\hline rmsea & $\leq .08$ & .08 \\
\hline cfi & Cercano a .95 & .91 \\
\hline srmr & $<0.05$ & .03 \\
\hline
\end{tabular}

El modelo quedó conformado por dos factores y 28 reactivos, que corresponden a ocho competencias (ver tabla 6). En comparación con el modelo original, se eliminó la competencia "definir criterios y actividades de evaluación y acreditación del curso" (García-Cabrero et al., 2018, p. 354).

Tabla 6. Estructura final del CECDL

\begin{tabular}{|c|c|c|}
\hline Dimensiones & Competencias & Ítems \\
\hline \multirow{7}{*}{$\begin{array}{l}\text { Previsión del } \\
\text { proceso de } \\
\text { enseñanza y } \\
\text { aprendizaje }\end{array}$} & \multirow{2}{*}{$\begin{array}{l}\text { Plantear el enfoque de la } \\
\text { asignatura }\end{array}$} & $\begin{array}{l}\text { 1. Estableció la relación de la asignatura con el } \\
\text { campo profesional. }\end{array}$ \\
\hline & & $\begin{array}{l}\text { 2. Estableció las metas de aprendizaje para la } \\
\text { asignatura. }\end{array}$ \\
\hline & \multirow{4}{*}{$\begin{array}{l}\text { Planear el curso de la } \\
\text { asignatura }\end{array}$} & $\begin{array}{l}\text { 3. Seleccionó materiales digitales pertinentes } \\
\text { en contenido para las actividades de } \\
\text { aprendizaje. }\end{array}$ \\
\hline & & $\begin{array}{l}\text { 4. Estableció al inicio del curso un cronograma } \\
\text { detallado de actividades (por ejemplo, } \\
\text { períodos de trabajo, fechas de entrega de } \\
\text { tareas individuales y grupales, exámenes). }\end{array}$ \\
\hline & & $\begin{array}{ll}\text { 5. } & \text { Acordó al inicio de la asignatura las } \\
\text { reglas de interacción en medios digitales } \\
\text { sustentadas en valores universales. }\end{array}$ \\
\hline & & $\begin{array}{l}\text { 6. Definió las reglas de trabajo para evitar el } \\
\text { plagio. }\end{array}$ \\
\hline & $\begin{array}{l}\text { Diseñar experiencias de } \\
\text { aprendizaje }\end{array}$ & $\begin{array}{l}\text { 7. Presentó en cada unidad instrucciones } \\
\text { detalladas para su desarrollo (por ejemplo, } \\
\text { propósitos de las actividades, dinámicas de } \\
\text { trabajo). }\end{array}$ \\
\hline
\end{tabular}




\begin{tabular}{|c|c|c|}
\hline Dimensiones & Competencias & Ítems \\
\hline & $\begin{array}{l}\text { Demostrar dominio } \\
\text { amplio de uso y } \\
\text { selección de TIC } \\
\text { pertinentes para la } \\
\text { enseñanza aprendizaje }\end{array}$ & $\begin{array}{l}\text { 8. Incluye en las actividades el uso de } \\
\text { Tecnologías de la Información y la } \\
\text { Comunicación (videos, wikis, blogs, } \\
\text { aplicaciones web, software) acordes a mis } \\
\text { posibilidades de aprendizaje. }\end{array}$ \\
\hline & $\begin{array}{l}\text { Utilizar formas de } \\
\text { comunicación adecuadas } \\
\text { para apoyar el trabajo } \\
\text { académico }\end{array}$ & 23. Hace un correcto uso del lenguaje. \\
\hline & $\begin{array}{l}\text { Utilizar formas } \\
\text { adecuadas para } \\
\text { valorar los procesos de } \\
\text { enseñanza, aprendizaje } \\
\text { autorregulado y } \\
\text { colaborativo en línea, así } \\
\text { como su impacto }\end{array}$ & $\begin{array}{l}\text { 26. Respeta los criterios de evaluación } \\
\text { presentados al inicio del curso. }\end{array}$ \\
\hline \multirow{8}{*}{$\begin{array}{l}\text { Conducción } \\
\text { y Evaluación } \\
\text { del proceso de } \\
\text { enseñanza y } \\
\text { aprendizaje }\end{array}$} & \multirow{7}{*}{$\begin{array}{l}\text { Gestionar la progresión } \\
\text { de los aprendizajes }\end{array}$} & $\begin{array}{l}\text { 9. Implementa actividades de aprendizaje } \\
\text { acordes con los propósitos de la asignatura. }\end{array}$ \\
\hline & & $\begin{array}{l}\text { 10. Facilita los materiales necesarios para } \\
\text { desarrollar los conocimientos propuestos en } \\
\text { la asignatura. }\end{array}$ \\
\hline & & $\begin{array}{l}\text { 11. Promueve el desarrollo de las actitudes } \\
\text { señaladas en la asignatura. }\end{array}$ \\
\hline & & $\begin{array}{l}\text { 12. Promueve la aplicación de los aprendizajes } \\
\text { en la solución de problemas reales. }\end{array}$ \\
\hline & & 13. Aclara las dudas de forma oportuna. \\
\hline & & $\begin{array}{l}\text { 14. Ajusta las actividades de aprendizaje } \\
\text { de acuerdo con las necesidades de los } \\
\text { estudiantes. }\end{array}$ \\
\hline & & $\begin{array}{l}\text { 15. Proporciona retroalimentación de forma } \\
\text { personalizada a través de la plataforma. }\end{array}$ \\
\hline & $\begin{array}{l}\text { Diseñar experiencias de } \\
\text { aprendizaje }\end{array}$ & $\begin{array}{l}\text { 16. Estructura actividades de aprendizaje } \\
\text { que propician la colaboración entre los } \\
\text { estudiantes. }\end{array}$ \\
\hline
\end{tabular}




\begin{tabular}{|c|c|c|}
\hline Dimensiones & Competencias & Ítems \\
\hline & \multirow{6}{*}{$\begin{array}{l}\text { Llevar a cabo la } \\
\text { interacción didáctica } \\
\text { orientada a incrementar } \\
\text { la motivación y las } \\
\text { expectativas de } \\
\text { resultados }\end{array}$} & $\begin{array}{l}\text { 17. Utiliza materiales digitales en diversos } \\
\text { formatos para enriquecer la experiencia de } \\
\text { aprendizaje. }\end{array}$ \\
\hline & & $\begin{array}{l}\text { 18. Verifica que los materiales digitales } \\
\text { seleccionados para cada actividad de } \\
\text { aprendizaje estén accesibles de forma } \\
\text { oportuna. }\end{array}$ \\
\hline & & $\begin{array}{l}\text { 19. Orienta a los estudiantes cuando existen } \\
\text { dudas o problemas con el manejo de } \\
\text { las Tecnologías de la Información y la } \\
\text { Comunicación (por ejemplo, aplicaciones } \\
\text { web y la plataforma) que se utilizan en el } \\
\text { curso. }\end{array}$ \\
\hline & & $\begin{array}{l}\text { 20. Fomenta que los estudiantes reflexionen } \\
\text { sobre sus experiencias de aprendizaje. }\end{array}$ \\
\hline & & 21. Fomenta el sentido de bienestar en el grupo. \\
\hline & & $\begin{array}{l}\text { 22. Realiza una evaluación diagnóstica al inicio } \\
\text { de la asignatura. }\end{array}$ \\
\hline & \multirow{2}{*}{$\begin{array}{l}\text { Utilizar formas de } \\
\text { comunicación adecuadas } \\
\text { para apoyar el trabajo } \\
\text { académico. }\end{array}$} & $\begin{array}{l}\text { 24. Mantiene comunicación continua con } \\
\text { los estudiantes a través de los medios de } \\
\text { interacción de la plataforma. }\end{array}$ \\
\hline & & $\begin{array}{l}\text { 25. Retroalimenta de forma oportuna el } \\
\text { desarrollo de las actividades de aprendizaje. }\end{array}$ \\
\hline & \multirow{2}{*}{$\begin{array}{l}\text { Utilizar formas } \\
\text { adecuadas para } \\
\text { valorar los procesos de } \\
\text { enseñanza, aprendizaje } \\
\text { autorregulado y } \\
\text { colaborativo en línea, así } \\
\text { como su impacto. }\end{array}$} & $\begin{array}{l}\text { 27. Realiza una evaluación final congruente con } \\
\text { las actividades de aprendizaje realizadas } \\
\text { durante el curso. }\end{array}$ \\
\hline & & $\begin{array}{l}\text { 28. Utiliza diversas estrategias para la } \\
\text { evaluación del curso acordes con los } \\
\text { objetivos de aprendizaje. }\end{array}$ \\
\hline
\end{tabular}

La Tabla 7 sintetiza las propiedades generales del CECDL.

Tabla 7. Propiedades del CECDL

\begin{tabular}{|l|l|}
\hline \multicolumn{1}{|c|}{ Propiedades } & \multicolumn{1}{c|}{ Descripción } \\
\hline Nombre del cuestionario & $\begin{array}{l}\text { Cuestionario de evaluación de la } \\
\text { Competencia Docente en Línea }\end{array}$ \\
\hline Población objetivo & Estudiantes inscritos en cursos en línea \\
\hline Usuarios & Directivos y docentes \\
\hline Uso de los resultados & Evaluación formativa \\
\hline Vía de administración & Plataforma en línea \\
\hline
\end{tabular}




\begin{tabular}{|c|c|c|}
\hline \multicolumn{2}{|l|}{ Dimensiones } & Ítems \\
\hline \multicolumn{2}{|c|}{ Previsión del proceso de enseñanza y aprendizaje } & 10 \\
\hline \multicolumn{2}{|c|}{ Conducción y Evaluación del proceso de enseñanza y aprendizaje } & 18 \\
\hline \multicolumn{2}{|l|}{ Total de ítems } & 28 \\
\hline Tipo de respuesta & \begin{tabular}{|l} 
Nunca \\
Pocas veces \\
Frecuentemente \\
Siempre
\end{tabular} & \\
\hline
\end{tabular}

\section{DISCUSIÓN Y CONCLUSIONES}

Este trabajo reconoce la necesidad de contar con instrumentos de evaluación de la competencia docente válidos, confiables y pertinentes al contexto educativo. Aun cuando los cuestionarios de evaluación de la docencia por los estudiantes tienen una larga tradición y uso generalizado, es incipiente la investigación y diseño de cuestionarios para evaluar la modalidad de enseñanza por competencias y, particularmente, la modalidad de enseñanza en línea (Benton y Cashin, 2014). De manera generalizada, se reconoce que las instituciones de educación superior, ante la necesidad de contar con instrumentos de evaluación de la docencia, recurren al desarrollo de instrumentos sin seguir un riguroso proceso de diseño que demuestre su validez. Lo cual repercute en minimizar la posibilidad de retroalimentar la práctica docente de forma coherente con el modelo pedagógico, modalidad de enseñanza y contexto institucional (RIIED, 2008).

$\mathrm{Al}$ incorporar los principios de enseñanza por competencias de la modalidad en línea, en la elaboración de los instrumentos de evaluación docente; se busca congruencia entre la evaluación y las demandas institucionales. Al mismo tiempo, la utilización de metodologías robustas para la acumulación de evidencias de validez de constructo abona a la validación teórica del mismo. De esta manera, los puntajes derivados de los cuestionarios representarán información válida para la retroalimentación de la práctica docente y la toma de decisiones.

El desarrollo del CECDL permitió operacionalizar en elementos medibles por los estudiantes los planteamientos teóricos de modelos propios de la modalidad de enseñanza en línea y por competencias integrados en el MECDL (García-Cabrero et al., 2018). Su diseño sellevó a cabo desde la perspectiva de evaluación de competencias por medio de indicadores, que asume que es posible el desglose de las competencias para su evaluación (Denyer, Furnemont, Poulain y Vanloubbeeck, 2007). Los resultados corroboraron el fundamento teórico del instrumento con medidas aceptables de validez. Por tanto, se superó una de las recurrentes limitaciones de los instrumentos de evaluación de la docencia que es su escasa fundamentación teórica y la exigua utilización de métodos psicométricos (Oon, Spencer y Chun, 2017). 
El CECDL fue diseñado para ser utilizado de forma institucional en una universidad pública mexicana con el fin de retroalimentar la práctica docente, permite interpretaciones válidas y confiables, por lo tanto, se recomienda su aplicación. Con ello, el objetivo de este trabajo se cumplimentó.

Si bien, la investigación sobre los cuestionarios de opinión de los estudiantes ha demostrado su validez de constructo y confiabilidad (Linse, 2017) persiste un amplio cuestionamiento sobre el uso de los puntajes, la interpretación de los resultados y sus consecuencias (Benton y Cashin, 2014), lo que alude al proceso de validez relacionado con el impacto en la mejora de los procesos de enseñanza y aprendizaje (validez de consecuencias). Por lo que cabe resaltar la persistente necesidad de vincular los resultados de la evaluación a estrategias de formación permanente ad hoc a las condiciones del profesorado.

\section{Agradecimientos}

Este trabajo fue realizado gracias al financiamiento otorgado al proyecto "Desarrollo y Validación de un Modelo de Evaluación de Competencias Docentes en Línea en Educación Superior" por el Programa para el Desarrollo Profesional Docente en 2015. Asimismo, se reconoce el valioso apoyo otorgado por el Centro de Educación Abierta y a Distancia- UABC.

\section{REFERENCIAS}

Arbaugh, J. B., Cleveland-Innes, M., Diaz, S. R., Garrison, R., Ice, P., Richardson, J. C., y Swan, K. P. (2008). Developing a community of inquiry instrument: Testing a measure of Community of Inquiry framework using a multi-institutional sample. Internet and Higher Education, 11,133-136. https://doi.org/10.1016/j. iheduc.2008.06.003

Bakieva, M., Jornet, J., González-Such, J., y Leyva, Y. (2018). Colegialidad docente: evidencias de validación a partir del análisis realizado por comités de expertos acerca del instrumento para autoevaluación docente en España y México. Estudios sobre Educación, 34, 99127. https://doi.org/10.15581/004.34.99$\underline{127}$

Baldwin, S., y Trespalacios, J. (2017). Evaluation instruments and good practices in online education. Online Learning,
21(2). http://dx.doi.org/10.24059/olj. v21i2.913

Bangert, A. W. (2008). The Development and Validation of the Student Evaluation of Online Teaching Effectiveness. Computers in the Schools, 25 (1-2), 25-47. https:// doi.org/10.1080/07380560802157717

Benton, S. L., y Cashin, W. E. (2014). Student ratings of instruction in college and university courses. En M. B. Paulsen, (Ed.), Higher Education: Handbook of Theory and Research, Volume 29 (279326). Springer Science+Business Media Dordrecht. https://doi.org/10.1007/97894-017-8005-6 7

Chickering, A. W., y Gamson, Z. F. (1987). Seven principles for good practice in undergraduate education. $A A H E$ Bulletin, 3-7. Recuperado de https://eric. ed.gov/?id=ED282491 
Cleveland-Innes, M., y Campbell, P. (2012). Emotional presence, learning, and the online learning environment. The International Review of Research in Open and Distributed Learning, 13(4), 269-292. https://doi.org/10.19173/irrodl. v13i4.1234

Darwin, S. (2017). What contemporary work are student ratings actually doing in higher education? Studies in Educational Evaluation, 54, 13-21. https://dx.doi. org/10.1016/j.stueduc.2016.08.002

Denyer, M., Furnemont, J., Poulain, R., y Vanloubbeeck, G. (2007). Las competencias en la educación, un balance. México: Fondo de Cultura Económica.

Domínguez-Lara, S. (2018). Fiabilidad y alfa ordinal. Actas Urológicas Españolas, 42(2), 140-141. https://doi.org/10.1016/j. acuro.2017.07.002

Finch, W. H., y French, B. F. (2019). Exploratory and Confirmatory Factor Analysis. En Educational and Psychological Measurement (135-169). Routledge.

García-Aretio, L. (2014). Bases, tendencias y futuro de la educación a distancia en la sociedad digital. España: Síntesis.

García-Cabrero, B., Luna, E., Ponce, S., Cisneros-Cohemour, E., Cordero, G., y Espinoza, J. (2018). Las competencias docentes en entornos virtuales: un modelo para su evaluación. RIED. Revista Iberoamericana de Educación a Distancia, 21(1), 343-365. https://doi. org/10.5944/ried.21.1.18816

Garrison, D. R., Anderson, T., y Archer, W. (2000). Critical Inquiry in a Text-Based Environment: Computer Conferencing in Higher Education. The Internet and Higher Education, 2(2), 87105. Recuperado de http://auspace. athabascau.ca/bitstream/2149/739/1/ critical inquiry in a text.pdf

Hornstein, A. (2017). Student evaluations of teaching are an inadequate assessment tool for evaluating faculty performance.
Cogent Education, 4(1). https://doi. org/10.1080/2331186X.2017.1304016

Laurillard, D. (2002). Rethinking teaching for the knowledge society. EDUCAUSE review, 37(1), 16-24. Recuperado de https://www.educause.edu/ir/library/ pdf/ffpiu017.pdf

Linacre, J.M. (2002). What do infit and outfit, mean-square and standardized mean? Rasch Measurement Transactions, 16(2), 878. Recuperado de https://www.rasch. org/rmt/rmt162f.htm

Linse, A. R. (2017). Interpreting and using student ratings data: Guidance for faculty serving as administrators and on evaluation committees. Studies in Educational Evaluation, 54, 94106. http://dx.doi.org/10.1016/j. stueduc.2016.12.004

Luna, E., Ponce, S., Cordero, G., y CisnerosCohernour, E. (2018). Marco para evaluar las condiciones institucionales de la enseñanza en línea. Revista Electrónica de Investigación Educativa, 2O(2), 1-14. https://doi.org/10.24320/ redie.2018.20.2.2072

Mauri, T., y Onrubia, J. (2008). El profesor en entornos virtuales: condiciones, perfil y competencias. En C. Coll y C. Monereo, (Eds.), Psicología de la educación virtual (132-152). Morata.

McClary, J. (2013). Factor in High quality distance Learning Courses. Online Journal of Distance Learnin Administration, 16 (2), 230-256. Recuperado de https:// www.westga.edu/ distance/ojdla/ summer162/mcclary162.html

Mishra, P., y Koehler, M. J. (2006). Technological pedagogical content knowledge: a framework for teacher knowledge. Teachers College Record, 108(6), 1017-1054. Recuperado de https://www.learntechlib.org/p/99246/

Nasser-Abu, F. (2017). Guest editor introduction to special issue "Contemporary evaluation of teaching: hallenges and promises". 
Studies in Educational Evaluation, 54, 1-3. http://dx.doi.org/10.1016/j. stueduc.2017.02.002

Olpak, Y. Z., y Kilic, E. (2018). Examining the realiability and validity of a Turkish version of the community of inquiry survey. Online Learning, 22(1), 147-161. http://dx.doi.org/10.24059/olj.v22i1.990

Oon, P. T., Spencer, B., y Chun, S. K. (2017). Psychometric quality of a student evaluation of teaching survey in higher education. Asseessment \& Evaluation in Higher Education, 42(5), 788-800.

https://doi.org/10.1080/02602938.2016 .1193119

Ravenscroft, B., Luhanga, U., y King, B. (2017). Adapting Bangert's online teaching effectiveness evaluation tool to a Canadian context. Innovations in Education and Teaching International, 54(4), 355-363. https://doi.org/10.1080/ $\underline{14703297.2016 .1231618}$

Red Iberoamericana de Investigadores sobre la Evaluación de la Docencia [RIIED]. (2008). Reflexiones sobre el diseño y puesta en marcha de programas de evaluación de la docencia. Revista Iberoamericana de Evaluación Educativa, 1(3e), 163-168. Recuperado de http://www.rinace.net/riee/numeros/ vol1-num3 e/reflexiones.html

Sireci, S., y Faulkner-Bond, M. (2014). Validity evidence based on test content. Psicothema, 26(1), 100-107. https://doi. org/10.7334/psicothema2013.256

Smith, W. C., y Kubacka, K. (2017). The emphasis of student test scores in teacher appraisal systems. Education policy analysis archives, 25(86). https://doi. org/10.14507/epaa.25.2889

Tabatabaee-Yazdi, M., Motallebzadeh, K., Ashraf, H., y Baghaei, P. (2018). Development and Validation of a Teacher Success Questionnaire Using the Rasch Model. International Journal of Instruction, 11(2), 129-144. https://doi. org/10.12973/iji.2018.11210a

Verma, J. P. (2019). Application of Factor Analysis in Psychological Data. En Statistics and Research Methods in Psychology with Excel (567-588). Springer.

\section{PERFIL ACADÉMICO Y PROFESIONAL DE LOS AUTORES}

Edna Luna Serrano. Licenciada y maestra en psicología por la Universidad Nacional Autónoma de México (UNAM) y doctora en educación por la Universidad Autónoma de Sinaloa (México). Investigadora en el Instituto de Investigación y Desarrollo Educativo de la Universidad Autónoma de Baja California. Miembro del Sistema Nacional de Investigadores. Su línea de investigación es evaluación educativa, en particular evaluación de la docencia y evaluación de la formación profesional.

E-mail: ednalunaserrano@gmail.com

Alma Delia Hernández Villafaña. Licenciada en Ciencias de la Educación y Maestra en Ciencias Educativas por la Universidad Autónoma de Baja California, actualmente es estudiante del Doctorado en Ciencias Educativas en la mimas universidad. Su línea de investigación es evaluación de la docencia en entornos presenciales y virtuales.

E-mail: alma.delia.hernandez.villafana@gmail.com 
Dirección:

Instituto de Investigación y Desarrollo Educativo,

Universidad Autónoma de Baja California

Km. 103 Carretera Tijuana-Ensenada. CP 22830

Ensenada, Baja California, México

Fecha de recepción del artículo: 25/03/2020

Fecha de aceptación del artículo: 04/11/2020

Fecha de aprobación para maquetación: 21/04/2020 